\title{
Exact solutions for the LMG model Hamiltonian based on the Bethe ansatz ${ }^{\text {is }}$
}

\author{
Hiroyuki Morita ${ }^{\text {a }}$, Hiromasa Ohnishi ${ }^{\text {a }}$, João da Providência ${ }^{\text {b }}$ \\ Seiya Nishiyama ${ }^{\mathrm{a}, \mathrm{b}, *, 1}$ \\ a Department of Applied Science, Graduate School of Science, Kochi University, Kochi 780-8520, Japan \\ b Centro de Física Teórica, Universidade de Coimbra, 3000 Coimbra, Portugal
}

Received 12 March 2004; received in revised form 5 December 2005; accepted 5 January 2006

Available online 23 January 2006

\begin{abstract}
Exact solutions for the Lipkin-Meshkov-Glick (LMG) model Hamiltonian are obtained by solving the Bethe ansatz equation (BAE) which is derived from the variation equation based on the Bethe ansatz. Unlike Pan and Draayer, we do not use bosonization and infinite-dimensional algebra techniques. Consequently there are no restrictions on parameters specifying strengths of the interactions included in the LMG Hamiltonian. Thus, for all the regimes of the interaction parameters, we get the exact solutions for the LMG Hamiltonian by numerically solving the BAEs and give the numerical behaviour of an order parameter $\left\langle J_{x}^{2}\right\rangle$. (C) 2006 Elsevier B.V. All rights reserved.
\end{abstract}

\section{Introduction}

For the past four decades, an approach to the exact solution of the BCS model [1] proposed by Richardson has shed light on theoretical developments in wider fields of quantum many-body physics $[2,3]$. He has shown that it is possible to determine simultaneously the eigenvalues and

\footnotetext{
A preliminary version of this work has first been presented by H. Morita at the Workshop on Quantum Field Theory and its Application held at Yukawa Institute for Theoretical Physics, Kyoto, Japan, 5-8 August 2003.

* Corresponding author.

E-mail addresses: morita@cc.kochi-u.ac.jp (H. Morita), ohnishi@cc.kochi-u.ac.jp (H. Ohnishi), providencia@teor.fis.uc.pt (J. da Providência), nisiyama@teor.fis.uc.pt, nisiyama@cc.kochi-u.ac.jp, seikoaquarius@ybb.ne.jp (S. Nishiyama).

1 Permanent address: Department of Applied Science, Graduate School of Science, Kochi University, Kochi 780-8520, Japan.
} 
the eigenstate of the BCS Hamiltonian from the solution of the algebraic equations, called the Bethe ansatz equation (BAE) [4]. Very recently, his method for the exact solution of the BAE has attracted much theoretical attention, because the BAE is gradually recognized to enrich the mathematical structures of the solutions in connection with the Gaudin's model [5], the integrable models [6] and the conformal field theory [7]. Also in a practical way his method is expected to open a new field for the exploration of superconducting nature of ultrasmall metallic grains [8]. If his method is used, it is, however, not so easy to calculate the expectation value of the Hamiltonian. It may become easily tractable if we introduce auxiliary fermion operators in addition to the original fermion ones. To show the effectiveness of our method, we will apply it to the Lipkin-Meshkov-Glick (LMG) model [9].

The LMG model is also exactly soluble. Pan and Draayer (PD) showed that its exact solution can be constructed by the BAE [10]. It has often been used as a simple but important model to clarify essential features of various methods and approximations and to illustrate their validity and effectiveness [11,12]. The LMG Hamiltonian is given as

$$
\begin{aligned}
H= & \frac{\epsilon}{2} \sum_{i=1}^{n}\left(c_{i+}^{\dagger} c_{i+}-c_{i-}^{\dagger} c_{i-}\right)-\frac{V}{2} \sum_{i=1}^{n} \sum_{i^{\prime}=1}^{n}\left(c_{i+1}^{\dagger} c_{i^{\prime}+}^{\dagger} c_{i^{\prime}-} c_{i-}+c_{i-}^{\dagger} c_{i^{\prime}-}^{\dagger} c_{i^{\prime}+} c_{i+}\right) \\
& -\frac{W}{2} \sum_{i=1}^{n} \sum_{i^{\prime}=1}^{n}\left(c_{i+}^{\dagger} c_{i^{\prime}-}^{\dagger} c_{i^{\prime}+} c_{i-}+c_{i-}^{\dagger} c_{i^{\prime}+}^{\dagger} c_{i^{\prime}-} c_{i+}\right),
\end{aligned}
$$

where $c_{i \sigma}^{\dagger}\left(c_{i \sigma}\right)$ is a fermion creation (annihilation) operator of particle with the state $i$ and $\sigma$ $(\sigma=+,-)$. The integer parameter $n$ denotes the number of values, the quantum number $i$, can assume. For the case of $W=0$, analytical and numerical studies of this model had been made extensively. For example, see Ref. [12]. However, for the general case of all the regimes of the interaction-strengths $V$ and $W$, a search for analytical and numerical solutions has not been pursued. Only for the case of $W^{2} \geqslant V^{2}$, PD derived the BAE after bosonization and obtained analytical expressions for the exact solutions by a method of infinite-dimensional loop algebra but without central extension. They, however, could obtain no analytical solutions for $W^{2}<V^{2}$. This problem is not solved yet. But it can be solved without the use of bosonization or infinitedimensional algebra techniques, unlike the PD approach.

In this paper we derive the BAE to obtain exact solutions of the LMG model for the most general case including $W^{2}<V^{2}$. Departing from the PD approach standing on the affine Lie algebra $\widehat{s u}(1,1)$ for bosons but along a way similar to the Richardson's approach, we can easily get the BAE from a variational equation for the wavefunctions, if we introduce auxiliary fermion operators in addition to the original fermion operators. The BAE obtained here avoids the strong restriction governing the relations between parameters in the PDs BAE.

The paper is organized as follows: In Section 2, we express eigenstates of the LMG Hamiltonian as product forms of building blocks. In Section 3.1, the BAE and analytical expressions for the exact solutions of the LMG Hamiltonian are derived from the variational equation, for the case $n=$ even; A-series. For the case $n=$ even; B-series, they are given in Section 3.2. In Section 4, we illustrate the behaviour of the eigenvalue spectra and the order parameter $\left\langle J_{x}^{2}\right\rangle$, for all cases. Finally, in Section 5, we give a discussion and some concluding remarks. The solutions for the case $n=$ odd are given in Appendix A. 


\section{Construction of eigenstates by using the Bethe ansatz}

Let us introduce auxiliary fermion creation-annihilation operators $d_{i \sigma}^{\dagger}$ and $\mathrm{d}_{i \sigma}$ and use two kinds of auxiliary vacuums $|0\rangle\rangle$ satisfying $\left.d_{i \sigma}|0\rangle\right\rangle=0$ and $\mid$ vac $\left.\rangle\right\rangle$ defined by $\left.|v a c\rangle\right\rangle \equiv$ $\left.d_{n-}^{\dagger} \cdots d_{1-}^{\dagger}|0\rangle\right\rangle$, respectively. This is done in parallel to the usual manner that we introduce two kinds of ordinary vacuums as $c_{i \sigma}|0\rangle=0$ and $|\mathrm{vac}\rangle \equiv c_{n-}^{\dagger} \cdots c_{1-}^{\dagger}|0\rangle$. We further define pair operators $a_{i}^{\dagger}$ and $a_{i}$ as $a_{i}^{\dagger} \equiv c_{i+}^{\dagger} c_{i-} \otimes \mathbb{1}_{d}$ and $a_{i} \equiv c_{i-}^{\dagger} c_{i+} \otimes \mathbb{1}_{d}$, where $\mathbb{1}_{d}$ is a unit operator in the $d$-fermion space $|\cdots\rangle\rangle$ and $\mathbb{1}$ is the usual unit operator. We also introduce auxiliary pair operators $b_{i}^{\dagger}$ and $b_{i}$ as $b_{i}^{\dagger} \equiv d_{i+}^{\dagger} d_{i-}$ and $b_{i} \equiv d_{i-}^{\dagger} d_{i+}$. Then, we have the identities $a_{i}^{\dagger} a_{i}^{\dagger}=0$ and $b_{i}^{\dagger} b_{i}^{\dagger}=0$ and $a_{i}|\mathrm{Vac}\rangle=0$ and $\left.b_{i}|\mathrm{vac}\rangle\right\rangle=0$ where $\left.|\mathrm{Vac}\rangle \equiv|\mathrm{vac}\rangle \otimes|\mathrm{vac}\rangle\right\rangle$.

The eigenstates $|n\rangle$ of the LMG model with $2 n$ particle-states are spanned by linear combinations of the states $c_{n \sigma_{n}}^{\dagger} \cdots c_{1 \sigma_{1}}^{\dagger}|0\rangle(\sigma=+,-)$. To construct the eigenstates $|n\rangle$ in product forms of building blocks, it is convenient to introduce the following mapping $\rho$ :

$$
\left.\rho: c_{i \sigma}^{\dagger} \mapsto c_{i \sigma}^{\dagger} \otimes b_{i}^{\dagger}, \quad \rho: c_{i \sigma} \mapsto c_{i \sigma} \otimes b_{i}, \quad \rho:|0\rangle \mapsto|0\rangle \otimes|\mathrm{vac}\rangle\right\rangle .
$$

Then, we have an important mapping rule for mathematical manipulations,

$$
\begin{aligned}
& \left.\rho: c_{n \sigma_{n}}^{\dagger} \cdots c_{1 \sigma_{1}}^{\dagger}|0\rangle \mapsto\left[c_{n \sigma_{n}}^{\dagger} \cdots c_{1 \sigma_{1}}^{\dagger} \otimes b_{n}^{\dagger} \cdots b_{1}^{\dagger}\right][|0\rangle \otimes|\mathrm{vac}\rangle\rangle\right] \\
& \left.=\left[\left(\prod_{i} c_{i+}^{\dagger} c_{i-}\right) \otimes b_{n}^{\dagger} \cdots b_{1}^{\dagger}\right][|\mathrm{vac}\rangle \otimes|\mathrm{vac}\rangle\rangle\right] \\
& \left(c_{i \sigma_{i}}^{\dagger}=c_{i+}^{\dagger}\right) \\
& =\left[\left(\prod_{i} c_{i+}^{\dagger} c_{i-} \otimes \mathbb{1}\right)\left(\mathbb{1} \otimes b_{n}^{\dagger} \cdots b_{1}^{\dagger}\right)\right]|\mathrm{Vac}\rangle \\
& \left(c_{i \sigma_{i}}^{\dagger}=c_{i+}^{\dagger}\right) \\
& =\left[\prod_{i} a_{i}^{\dagger}\right]\left(\mathbb{1} \otimes b_{n}^{\dagger} \cdots b_{1}^{\dagger}\right)|\mathrm{Vac}\rangle \\
& \left(c_{i \sigma_{i}}^{\dagger}=c_{i+}^{\dagger}\right)
\end{aligned}
$$

Two kinds of eigenstates can be constructed, which are classified into an A-series and a B-series. The eigenstates belonging to the A-series are given by a linear combination of states containing even numbers of $(+)$ signs in configuration $\left\{\sigma_{1}, \ldots, \sigma_{n}\right\}$ and the B-series eigenstates with odd numbers of $(+)$ signs in the $\left\{\sigma_{1}, \ldots, \sigma_{n}\right\}$. From the above, any eigenstate belonging to A-series (B-series) can be mapped to a state expressed as a linear combination of statevectors which are generated by even (odd) numbers of $a_{i}^{\dagger}$ 's acting on $\left(\mathbb{1} \otimes b_{n}^{\dagger} \cdots b_{1}^{\dagger}\right)|\mathrm{Vac}\rangle$.

Define the state $|n\rangle_{\rho}$ through the mapping $\rho$ of the eigenstate $|n\rangle$ of the LMG model. Then, the state $|n\rangle_{\rho}$ can be expressed as the product form of building blocks $B_{v}, D$ and $A$ :

$$
\begin{aligned}
& |n\rangle_{\rho}= \begin{cases}\prod_{v=1}^{n^{\prime}} B_{v}|\mathrm{Vac}\rangle & \text { (for } \left.n=\text { even, } n^{\prime}=\frac{n}{2} ; \text { A-series }\right), \\
\prod_{\nu=1}^{n^{\prime \prime}} B_{v} D A|\mathrm{Vac}\rangle & \left(\text { for } n=\text { even, } n^{\prime \prime}=\frac{n}{2}-1 ; \text { B-series }\right), \\
& D \equiv \sum_{i=1}^{n}\left(\mathbb{1} \otimes b_{i}^{\dagger}\right), A \equiv \sum_{i=1}^{n} a_{i}^{\dagger}\left(\mathbb{1} \otimes b_{i}^{\dagger}\right),\end{cases} \\
& |n\rangle_{\rho}= \begin{cases}\prod_{\nu=1}^{n^{\prime \prime \prime}} B_{v} D|\mathrm{Vac}\rangle & \left(\text { for } n=\text { odd }, n^{\prime \prime \prime}=\frac{1}{2}(n-1) ; \text { A-series }\right), \\
\prod_{\nu=1}^{n^{\prime \prime \prime}} B_{v} A|\mathrm{Vac}\rangle & \text { (for } \left.n=\text { odd }, n^{\prime \prime \prime}=\frac{1}{2}(n-1) ; \text { B-series }\right) .\end{cases}
\end{aligned}
$$




\section{Solutions of the LMG model Hamiltonian}

\subsection{Solution for the case $n=$ even; $A$-series}

The state $|n\rangle_{\rho}$ in the upper case of (2.3) is expanded in terms of the states $c_{n \sigma_{n}}^{\dagger} \cdots c_{1 \sigma_{1}}^{\dagger}|0\rangle \otimes$ $\left.b_{n}^{\dagger} \cdots b_{1}^{\dagger}|\mathrm{vac}\rangle\right\rangle$ as

$$
\begin{aligned}
& \left.|n\rangle_{\rho}=\mathcal{N} \sum_{\sigma_{1}, \ldots, \sigma_{n}}^{\prime} \varphi\left(\sigma_{1}, \ldots, \sigma_{n}\right) c_{n \sigma_{n}}^{\dagger} \cdots c_{1 \sigma_{1}}^{\dagger}|0\rangle \otimes b_{n}^{\dagger} \cdots b_{1}^{\dagger}|\mathrm{vac}\rangle\right\rangle, \\
& \mathcal{N}=\left[\sum_{\sigma_{1}, \ldots, \sigma_{n}}\left|\varphi\left(\sigma_{1}, \ldots, \sigma_{n}\right)\right|^{2}\right]^{-1 / 2} \quad \text { (normalization factor) }
\end{aligned}
$$

where $\sum^{\prime}$ means the sum over all the possible terms with even number of $(+)$ sign in the configuration $\left\{\sigma_{1}, \ldots, \sigma_{n}\right\}$. The expansion coefficient $\varphi\left(\sigma_{1}, \ldots, \sigma_{n}\right)$ can be regarded as a wave function. From (2.3) and (3.1), we get the explicit form of the wave function in terms of $\Lambda_{v}^{1}$ and $\Lambda_{v}^{2}$ as

$$
\varphi\left(\sigma_{1}, \ldots, \sigma_{n}\right)=m !(n-m) ! \frac{1}{m^{\prime} !} \frac{1}{\left(n^{\prime}-m^{\prime}\right) !} \sum_{P} P \Lambda_{P_{1}}^{1} \cdots \Lambda_{P_{m^{\prime}}}^{1} \Lambda_{P_{m^{\prime}+1}}^{2} \cdots \Lambda_{P_{n^{\prime}}}^{2},
$$

where $m$ is the number of $(+)$ sign in the $\left\{\sigma_{1}, \ldots, \sigma_{n}\right\}$ and $m^{\prime}=m / 2$ and $\sum_{P} P$ is the sum over the $n^{\prime}$ ! permutations of the indices $1, \ldots, n^{\prime}$.

Since the mapping by $\rho$ is one-to-one as seen below,

$$
\begin{aligned}
\rho: & \sum_{\sigma_{1}, \ldots, \sigma_{n}}^{\prime} \varphi\left(\sigma_{1}, \ldots, \sigma_{n}\right) c_{n \sigma_{n}}^{\dagger} \cdots c_{1 \sigma_{1}}^{\dagger}|0\rangle \\
& \left.\mapsto \sum_{\sigma_{1}, \ldots, \sigma_{n}}^{\prime} \varphi\left(\sigma_{1}, \ldots, \sigma_{n}\right) c_{n \sigma_{n}}^{\dagger} \cdots c_{1 \sigma_{1}}^{\dagger}|0\rangle \otimes b_{n}^{\dagger} \cdots b_{1}^{\dagger}|\mathrm{vac}\rangle\right\rangle
\end{aligned}
$$

if we can get all the $\varphi\left(\sigma_{1}, \ldots, \sigma_{n}\right)$, the state $|n\rangle$ is completely determined. Giving only a number $m$, the corresponding wave function $\varphi$ can be solved independently on the configuration $\left\{\sigma_{1}, \ldots, \sigma_{n}\right\}$. Denote such a solution simply as $\varphi^{(m)}$. From the variation equation

$$
\frac{\delta\langle n|H-E| n\rangle}{\delta \varphi^{(m) *}\left(\sigma_{1}, \ldots, \sigma_{n}\right)}=\frac{\delta\langle n|H-E| n\rangle}{\delta \varphi^{(m)}\left(\sigma_{1}, \ldots, \sigma_{n}\right)}=0
$$

the relations among $\varphi^{(m)}\left(\sigma_{1}, \ldots, \sigma_{n}\right)$ for $m=0,2, \ldots, n$ are derived in the following form:

$$
\begin{aligned}
0=\{ & \left.-\left(n^{\prime}-m^{\prime}\right) \epsilon+m^{\prime} \epsilon-E\right\} \varphi^{(m)}\left(\sigma_{1}, \ldots, \sigma_{n}\right) \\
& -V \sum_{k} \sum_{\substack{l(k<l) \\
\left(\sigma_{k}=\sigma_{l}=-\right)}} \varphi^{(m+2)}\left(\sigma_{1}, \ldots,\left[-\sigma_{k}\right], \ldots,\left[-\sigma_{l}\right], \ldots, \sigma_{n}\right) \\
& -V \sum_{k} \sum_{\substack{l(k<l) \\
\left(\sigma_{k}=\sigma_{l}=+\right)}} \varphi^{(m-2)}\left(\sigma_{1}, \ldots,\left[-\sigma_{k}\right], \ldots,\left[-\sigma_{l}\right], \ldots, \sigma_{n}\right) \\
& -W \sum_{k} \sum_{\substack{l(k \neq l) \\
\left(\sigma_{k}=-\sigma_{l}\right)}} \varphi^{(m)}\left(\sigma_{1}, \ldots,\left[-\sigma_{k}\right], \ldots,\left[-\sigma_{l}\right], \ldots, \sigma_{n}\right)
\end{aligned}
$$

and its complex conjugate.

In the above, the pair $\left\{\left[-\sigma_{k}\right],\left[-\sigma_{l}\right]\right\}$ stands for values with opposite sign to those of the pair $\left\{\left[\sigma_{k}\right],\left[\sigma_{l}\right]\right\}$ in the original configuration $\left\{\sigma_{1}, \ldots, \sigma_{n}\right\}$. The second term, the third and the last in 
the right-hand side are given by sums over $\frac{1}{2}(n-m)(n-m-1)$ terms, $\frac{1}{2} m(m-1)$ terms and $m(n-m)$ terms, respectively.

Substitution of Eq. (3.2) into Eq. (3.5) for $m^{\prime}=0, \ldots, n^{\prime}$ leads to

$$
\begin{aligned}
& 0=\left\{-\left(n^{\prime}-m^{\prime}\right) \epsilon+m^{\prime} \epsilon-E\right\} \sum_{P} P\left[\prod_{\mu} \Lambda_{\mu}^{1} \prod_{\nu} \Lambda_{v}^{2}\right] \\
& -\frac{V}{2}(n-m)(m+1) \sum_{P} P\left[\Lambda_{P_{m^{\prime}+1}}^{1} \prod_{\mu} \Lambda_{\mu}^{1} \prod_{\substack{\nu \\
\nu \neq P_{m^{\prime}+1}}} \Lambda_{\nu}^{2}\right] \\
& -\frac{V}{2}(n-m+1) m \sum_{P} P\left[\Lambda_{P_{m^{\prime}}}^{2} \prod_{\substack{\mu \\
\mu \neq P_{m^{\prime}}}} \Lambda_{\mu}^{1} \prod_{\nu} \Lambda_{v}^{2}\right] \\
& -W m(n-m) \sum_{P} P\left[\prod_{\mu} \Lambda_{\mu}^{1} \prod_{\nu} \Lambda_{v}^{2}\right] \\
& =\sum_{P} P\left[\left[\left\{-\left(n^{\prime}-m^{\prime}\right) \epsilon+m^{\prime} \epsilon-E-W m(n-m)\right\} \Lambda_{P_{m^{\prime}}}^{1} \Lambda_{P_{m^{\prime}+1}}^{2}\right.\right. \\
& \left.-V\left(2 m^{\prime}+1\right)\left(n^{\prime}-m^{\prime}\right) \Lambda_{P_{m^{\prime}}}^{1} \Lambda_{P_{m^{\prime}+1}}^{1}-V m^{\prime}\left\{2\left(n^{\prime}-m^{\prime}\right)+1\right\} \Lambda_{P_{m^{\prime}}}^{2} \Lambda_{P_{m^{\prime}+1}}^{2}\right] \\
& \left.\times \prod_{\substack{\mu \\
\mu \neq P_{m^{\prime}}}} \Lambda_{\mu}^{1} \prod_{\substack{\nu \\
\nu \neq P_{m^{\prime}+1}}} \Lambda_{v}^{2}\right]
\end{aligned}
$$

where we have denoted the product symbols $\prod_{\mu=P_{1}}^{P_{m^{\prime}}}$ and $\prod_{\nu=P_{m^{\prime}+1}}^{P_{n^{\prime}}}$ as $\prod_{\mu}$ and $\prod_{\nu}$, respectively.

To derive explicitly the BAEs, we will consider the following two cases.

The case (1) of $W \neq \pm V$.

Let the forms of $\Lambda_{j}^{1}$ and $\Lambda_{j}^{2}$ be

$$
\Lambda_{j}^{1}=\frac{\beta_{1}}{1-b_{1} x_{j}}, \quad \Lambda_{j}^{2}=\frac{\beta_{2}}{1-b_{2} x_{j}} .
$$

We assume that the above sets of quantities $\left(b_{1}, b_{2}\right)$ and $\left(\beta_{1}, \beta_{2}\right)$ satisfy the relations

$$
\left.\begin{array}{l}
b_{1}=\beta_{1}^{2}, \quad b_{2}=\beta_{2}^{2} \\
\frac{\beta_{1}^{2}+\beta_{2}^{2}}{\beta_{1} \beta_{2}}=-\frac{2 W}{V}
\end{array}\right\}
$$

and that $x_{j}$ is a complex $c$-number to be determined. The second relation in (3.8) is very similar to the relation adopted by PD for preserving the affine Lie algebra $\widehat{s u}(1,1)$ without central extension [10]. Ours, however, avoids the strong restriction due to the affine Lie algebra. We also assume the eigenvalue $E$ to be

$$
E=-\frac{\beta_{1}^{2}+\beta_{2}^{2}}{\beta_{1}^{2}-\beta_{2}^{2}} n^{\prime} \epsilon+\left\{\frac{1}{\beta_{1} \beta_{2}}(n-1) V+\frac{2}{\beta_{1}^{2}-\beta_{2}^{2}} \epsilon\right\} \sum_{j=1}^{n^{\prime}} \frac{1}{x_{j}} .
$$


Substituting Eqs. (3.7), (3.8) and (3.9) into Eq. (3.6) for $m^{\prime}=0, \ldots, n^{\prime}$, it is rearranged as

$$
\begin{aligned}
0= & \sum_{P} P\left[\frac{1}{\beta_{2}} \sum_{i=1}^{m^{\prime}}(-V)\right. \\
& \times\left\{\sum_{k=1}^{n^{\prime}} \frac{4}{x_{k}-x_{i}}+\beta_{1} \Lambda_{i}^{1}+\beta_{2} \Lambda_{i}^{2}+\left((n-1)+\frac{2 \beta_{1} \beta_{2}}{\beta_{1}^{2}-\beta_{2}^{2}} \frac{\epsilon}{V}\right) \frac{1}{x_{i}}\right\} \\
& \times \prod_{\mu \neq i} \Lambda_{\mu}^{1} \prod_{\nu} \Lambda_{v}^{2}+\frac{1}{\beta_{1}} \sum_{j=m^{\prime}+1}^{n^{\prime}}(-V) \\
& \times\left\{\sum_{k=1}^{n^{\prime}} \sum_{(k \neq j)} \frac{4}{x_{k}-x_{j}}+\beta_{1} \Lambda_{j}^{1}+\beta_{2} \Lambda_{j}^{2}+\left((n-1)+\frac{2 \beta_{1} \beta_{2}}{\beta_{1}^{2}-\beta_{2}^{2}} \frac{\epsilon}{V}\right) \frac{1}{x_{j}}\right\} \\
& \left.\times \prod_{\mu} \Lambda_{\mu}^{1} \prod_{\substack{v \\
\nu \neq j}} \Lambda_{v}^{2}\right]
\end{aligned}
$$

in which, to our surprise, there appears a common term contained in the curly blackest. Then, Eq. (3.10) is satisfied if the common term vanishes. Thus, we reach the BAE to determine $x_{j}$ $\left(j=1, \ldots, n^{\prime}\right)$ as

$$
\left\{(n-1)+\frac{2 \beta_{1} \beta_{2}}{\beta_{1}^{2}-\beta_{2}^{2}} \frac{\epsilon}{V}\right\} \frac{1}{x_{j}}+\frac{\beta_{1}^{2}}{1-\beta_{1}^{2} x_{j}}+\frac{\beta_{2}^{2}}{1-\beta_{2}^{2} x_{j}}=-\sum_{k=1(k \neq j)}^{n^{\prime}} \frac{4}{x_{k}-x_{j}},
$$

through which the eigenvalue and the corresponding eigenstate are given by (3.9) and (3.7).

The case (2) of $W= \pm V$.

In this case we assume

$$
\begin{aligned}
& b_{2}=-b_{1}, \quad \beta_{2}= \pm \beta_{1}\left\{\begin{array}{l}
\text { upper sign for } W=V, \\
\text { lower sign for } W=-V,
\end{array}\right. \\
& E=\mp \frac{V}{2} n^{2} \pm \frac{V}{2} n+\epsilon b_{1} \sum_{j=1}^{n^{\prime}} x_{j} .
\end{aligned}
$$

Using these relations, Eq. (3.6) for $m^{\prime}=0, \ldots, n^{\prime}$ is transformed to

$$
\begin{aligned}
0= & \sum_{P} P\left[\frac{\beta_{2}}{b_{2}} \sum_{i=1}^{m^{\prime}}(-V)\left(\sum_{k=1(k \neq i)}^{n^{\prime}} \frac{4}{x_{k}-x_{i}}+\frac{b_{1}}{\beta_{1}} \Lambda_{i}^{1}+\frac{b_{2}}{\beta_{2}} \Lambda_{i}^{2} \pm b_{1} \frac{\epsilon}{V}\right) \prod_{\substack{\mu \neq i \\
\mu \neq i}} \Lambda_{\mu}^{1} \prod_{\nu} \Lambda_{v}^{2}\right. \\
& \left.+\frac{\beta_{1}}{b_{1}} \sum_{j=m^{\prime}+1}^{n^{\prime}}(-V)\left(\sum_{k=1(k \neq j)}^{n^{\prime}} \frac{4}{x_{k}-x_{j}}+\frac{b_{1}}{\beta_{1}} \Lambda_{j}^{1}+\frac{b_{2}}{\beta_{2}} \Lambda_{j}^{2} \pm b_{1} \frac{\epsilon}{V}\right) \prod_{\mu} \Lambda_{\mu}^{1} \prod_{\substack{\nu \\
v \neq j}} \Lambda_{v}^{2}\right],
\end{aligned}
$$


from which we get the BAE

$$
\pm b_{1} \frac{\epsilon}{V}+\frac{b_{1}}{1-b_{1} x_{j}}+\frac{b_{2}}{1-b_{2} x_{j}}=-\sum_{k=1(k \neq j)}^{m^{\prime}} \frac{4}{x_{k}-x_{j}} .
$$

\subsection{Solution for the case $n=$ even; $B$-series}

The state $|n\rangle_{\rho}$ in the lower case of (2.3) has as the same expression as (3.1) but the sum is made for all the possible terms with odd numbers of $(+)$ signs in the configuration $\left\{\sigma_{1}, \ldots, \sigma_{n}\right\}$. Then, we have

$$
\begin{aligned}
& \varphi^{(m)}\left(\sigma_{1}, \ldots, \sigma_{n}\right) \\
& \quad=m !(n-m) ! \frac{1}{m^{\prime \prime} !} \frac{1}{\left(n^{\prime \prime}-m^{\prime \prime}\right) !} \sum_{P} P \Lambda_{P_{1}}^{1} \cdots \Lambda_{P_{m^{\prime \prime}}}^{1} \Lambda_{P_{m^{\prime \prime}+1}}^{2} \cdots \Lambda_{P_{n^{\prime \prime}}}^{2},
\end{aligned}
$$

where $m^{\prime \prime}=(m-1) / 2$ and $\sum_{P} P$ is the sum over $n^{\prime \prime}$ ! permutations of the indices $1, \ldots, n^{\prime \prime}$. Applying again the variation equation (3.4), the wave functions $\varphi^{(m)}\left(\sigma_{1}, \ldots, \sigma_{n}\right)$ are required to satisfy the same equation as (3.5) but for $m=1,3, \ldots, n-1$. Substitution of (3.16) into (3.5) for $m^{\prime \prime}=0, \ldots, n^{\prime \prime}$ leads to

$$
\begin{aligned}
0= & \sum_{P} P\left[\left[\left\{-\left(n^{\prime}-m^{\prime}\right) \epsilon+m^{\prime} \epsilon-E-W m(n-m)\right\} \Lambda_{P_{m^{\prime \prime}}}^{1} \Lambda_{P_{m^{\prime \prime}+1}}^{2}\right.\right. \\
& \left.-V\left(2 m^{\prime \prime}+3\right)\left(n^{\prime \prime}-m^{\prime \prime}\right) \Lambda_{P_{m^{\prime \prime}}}^{1} \Lambda_{P_{m^{\prime \prime}+1}}^{1}-V m^{\prime \prime}\left\{2\left(n^{\prime \prime}-m^{\prime \prime}\right)+3\right\} \Lambda_{P_{m^{\prime \prime}}}^{2} \Lambda_{P_{m^{\prime \prime}+1}}^{2}\right] \\
& \left.\times \prod_{\substack{\mu^{\prime \prime} \\
\mu^{\prime \prime} \neq P_{m^{\prime \prime}}}} \Lambda_{\mu^{\prime \prime}}^{1} \prod_{\substack{v^{\prime \prime} \\
v^{\prime \prime} \neq P_{m^{\prime \prime}+1}}} \Lambda_{v^{\prime \prime}}^{2}\right],
\end{aligned}
$$

where we have denoted the product symbols $\prod_{\mu^{\prime \prime}=P_{1}}^{P_{m^{\prime \prime}}}$ and $\prod_{v^{\prime \prime}=P_{m^{\prime \prime}+1}}^{P_{n^{\prime \prime}}}$ as $\prod_{\mu^{\prime \prime}}$ and $\prod_{v^{\prime \prime}}$, respectively.

The case (1) of $W \neq \pm V$.

Along a way similar to the one in the previous subsection, using Eq. (3.7) and the assumptions (3.8) and

$$
E=-\frac{\beta_{1}^{2}+\beta_{2}^{2}}{\beta_{1}^{2}-\beta_{2}^{2}} n^{\prime \prime} \epsilon-W(n-1)+\left\{\frac{1}{\beta_{1} \beta_{2}}(n-1) V+\frac{2}{\beta_{1}^{2}-\beta_{2}^{2}} \epsilon\right\} \sum_{j=1}^{n^{\prime \prime}} \frac{1}{x_{j}},
$$

then, the BAE for $j=1, \ldots, n^{\prime \prime}$ becomes

$$
\left\{(n-1)+\frac{2 \beta_{1} \beta_{2}}{\beta_{1}^{2}-\beta_{2}^{2}} \frac{\epsilon}{V}\right\} \frac{1}{x_{j}}+\frac{3 \beta_{1}^{2}}{1-\beta_{1}^{2} x_{j}}+\frac{3 \beta_{2}^{2}}{1-\beta_{2}^{2} x_{j}}=-\sum_{k=1(k \neq j)}^{n^{\prime \prime}} \frac{4}{x_{k}-x_{j}} .
$$

The case (2) of $W= \pm V$.

Using Eq. (3.7) and the assumptions (3.12) and

$$
E=\mp \frac{V}{2} n^{2} \pm \frac{V}{2} n+\epsilon b_{1} \sum_{j=1}^{n^{\prime \prime}} x_{j}
$$


then, the BAE for $j=1, \ldots, n^{\prime \prime}$ becomes

$$
\pm b_{1} \frac{\epsilon}{V}+\frac{3 b_{1}}{1-b_{1} x_{j}}+\frac{3 b_{2}}{1-b_{2} x_{j}}=-\sum_{k=1(k \neq j)}^{n^{\prime \prime}} \frac{4}{x_{k}-x_{j}} .
$$

The solutions for $n=$ odd are given in Appendix A.

\section{Behaviour of eigenvalue spectra and $\left\langle J_{x}^{2}\right\rangle$}

Let us introduce a dimensionless interaction-strength parameter $v \equiv V / \epsilon$ and a dimensionless eigenvalue $\mathcal{E} \equiv E / \epsilon$. For the case (2) of $W=V$, in all the regimes of $v$, the eigenvalue and the eigenstate are described in the previous section. Now we consider two limiting cases; One is $v \rightarrow 0$ and the other $v \rightarrow \infty$.

For the limiting case of $v \rightarrow 0$, the eigenvalue $\mathcal{E}$ of $n$ particles system is given as follows:

$$
\begin{cases}\mathcal{E}=-\frac{n}{2},-\frac{n}{2}+2,-\frac{n}{2}+4, \ldots & \text { (A-series), } \\ \mathcal{E}=-\frac{n}{2}+1,-\frac{n}{2}+3, \ldots & \text { (B-series). }\end{cases}
$$

The corresponding eigenstate is given by the usual eigenstate of angular momentum, $|J, M\rangle$

$$
\left|J\left(=\frac{n}{2}\right), M\right\rangle, \quad \begin{cases}M=-J,-J+2,-J+4, \ldots & \text { (A-series), } \\ M=-J+1,-J+3, \ldots & \text { (B-series). }\end{cases}
$$

For the strong coupling limit of $v \rightarrow \infty$, the eigenvalue $\mathcal{E}$ is given as follows:

$$
\mathcal{E}=\left(-\frac{1}{2} v\right) v^{2}-\left(-\frac{1}{2} v\right) n, \quad\left\{\begin{array}{c}
\Omega=n^{\prime}(n=\text { even }), \text { A-series, I; } \\
n^{\prime \prime}(n=\text { even }), \text { B-series, II, } \\
\Omega=n^{\prime \prime \prime}(n=\text { odd }), \text { A-series, III; B-series, IV, }
\end{array}\right.
$$

where $v=n-2 r$ and $r=0,1, \ldots, \Omega$. Due to (4.3), it turns out that for $n=$ even the eigenvalue becomes always two-fold degenerate except the level of $v=0$ and for $n=$ odd the eigenvalue is always two-fold degenerate.

Suppose $b_{1}=1$ and $b_{2}=-1$. The BAEs in Section 3 and in Appendix A becomes:

$$
\frac{1}{v}+\frac{s_{1}}{1-x_{j}}-\frac{s_{2}}{1+x_{j}}=-\sum_{k=1(k \neq j)}^{\Omega} \frac{4}{x_{k}-x_{j}}, \quad \begin{cases}s_{1}=1(3), & s_{2}=1(3), \text { I (II), } \\ s_{1}=1(3), & s_{2}=3(1), \text { III (IV) }\end{cases}
$$

For $z_{j}=\frac{x_{j}}{2 v}(j=1, \ldots, \Omega)$, Eq. (4.4) becomes

$$
1-\sum_{k=1(k \neq j)}^{\Omega} \frac{2}{z_{j}-z_{k}}-\frac{1}{2} \frac{s_{1}}{z_{j}-\frac{1}{2 v}}-\frac{1}{2} \frac{s_{2}}{z_{j}+\frac{1}{2 v}}=0 .
$$

In the above equation when $v \rightarrow \infty$, we can neglect the order $O(1 / v)$. Suppose the $(\Omega-r)$ variables, $z_{r+1}, \ldots, z_{\Omega}$, are in $O(1 / v)$ and the other $r$ variables, $z_{1}, \ldots, z_{r}$, are in $O(1)$. Then, in the limit of $v \rightarrow \infty$, a part of the solutions of the BAE, $z_{1}, \ldots, z_{r}$, satisfies

$$
1-\sum_{k=1(k \neq j)}^{r} \frac{2}{z_{j}-z_{k}}-\frac{1}{z_{j}}\left\{\frac{1}{2}\left(s_{1}+s_{2}\right)+2(\Omega-r)\right\}=0 \quad(j=1, \ldots, r) .
$$


The solutions of these equations are given by the zeros of the Sonine polynomial [13,14], determined by $L_{r}^{(v)}\left(\frac{x_{j}}{2 v}\right)=0$. The remaining solution is governed by an equation similar to (4.4) but without a term $1 / v$.

We are now in the stage to investigate the behaviour of the order parameter of the LMG model within the framework of the BAE. Let us introduce an angular momentum operator $J_{x}=\frac{1}{2}\left(J_{+}+J_{-}\right), J_{+} \equiv \sum_{j=1}^{n} c_{j+}^{\dagger} c_{j-}=J_{-}^{\dagger}$. For $W=V$, as an example, the order parameter $\left\langle J_{x}^{2}\right\rangle \equiv\left\langle n\left|J_{x}^{2}\right| n\right\rangle$ for the case $n=$ even belonging to the A-series is computed to be

$$
\begin{aligned}
J_{x}^{2}|n\rangle= & \frac{1}{2 \sqrt{N}} \sum_{k=0}^{\frac{n}{2}}\left\{\left(\begin{array}{c}
2 k \\
2
\end{array}\right) \varphi^{(2 k-2)}+\left(\begin{array}{c}
n-2 k \\
2
\end{array}\right) \varphi^{(2 k+2)}+\left(\frac{n}{2}+2 n k-4 k^{2}\right) \varphi^{(2 k)}\right\} \\
& \times \sum_{\substack{\sigma_{1}, \ldots, \sigma_{n} \\
(m=2 k)}} c_{n \sigma_{n}}^{\dagger} \cdots c_{1 \sigma_{1}}^{\dagger}|0\rangle,
\end{aligned}
$$

where $m$ is again the number of (+) sign in the configuration $\left\{\sigma_{1}, \ldots, \sigma_{n}\right\}$ and $N$ is given soon below. From (4.7) we have the final forms of $\left\langle J_{x}^{2}\right\rangle$ and the $N$ as

$$
\begin{aligned}
\left\langle J_{x}^{2}\right\rangle= & \frac{1}{2 N}\left\{\sum_{k=1}^{\frac{n}{2}}\left(\begin{array}{c}
n \\
2 k
\end{array}\right)\left(\begin{array}{c}
2 k \\
2
\end{array}\right) \varphi^{(2 k)^{*}} \varphi^{(2 k-2)}+\sum_{k=0}^{\frac{n}{2}-1}\left(\begin{array}{c}
n \\
2 k
\end{array}\right)\left(\begin{array}{c}
n-2 k \\
2
\end{array}\right) \varphi^{(2 k)^{*}} \varphi^{(2 k+2)}\right. \\
& \left.+\sum_{k=0}^{\frac{n}{2}}\left(\begin{array}{c}
n \\
2
\end{array}+2 n k-4 k^{2}\right)\left(\begin{array}{c}
n \\
2 k
\end{array}\right) \varphi^{(2 k)^{*}} \varphi^{(2 k)}\right\}, \\
N=\mathcal{N}^{-2}= & \sum_{k=0}^{\frac{n}{2}}\left(\begin{array}{c}
n \\
2 k
\end{array}\right) \varphi^{(2 k)^{*}} \varphi^{(2 k)} .
\end{aligned}
$$

At a finite range of $v$, the eigenvalue spectra, a term $\frac{W}{2} n$ having been subtracted, exhibit intermediate behaviour between those obtained at the two limiting cases mentioned above. In Fig. 1 , they are plotted versus the parameter $v$, at $0<v<0.6$ for $n=10$ where the symbols A0, etc., in the figure box mean the levels for the A-series, with $r=0$, etc. The behaviour is exactly the same as that drawn by PD [10] except that the interaction-strength parameter is attractive, i.e., $-v(v>0)$. In Fig. 2, we show the order parameter $\left\langle J_{x}^{2}\right\rangle$ as a function of $v$. It should be noticed that its behaviour has strong resemblance to the one shown in Ref. [12] which has been obtained by the resonating mean-field approximation [15].

As pointed out in the previous section, only for the case of $W^{2} \geqslant V^{2}$, PD have derived the BAE and obtained the analytical expressions for the exact solutions by employing the techniques of bosonization and infinite-dimensional loop algebra without central extension [10]. They, however, could obtain no analytical expressions for $W^{2}<V^{2}$. This problem is not still solved yet. In this paper with the use of the auxiliary fermion operator method, we have derived the BAE to get the exact solutions of the LMG model for the most general regime including $W^{2}<V^{2}$. For the case (1) of $W \neq V$, the behaviour of the eigenvalues as functions of $v$ for $n=10$ with $W=0-10 \mathrm{~V}$ is presented in Figs. 3-6. In particular, Fig. 4 gives an illustration of the solution belonging to the case of $W^{2}<V^{2}$. In Figs. 5 and 6, which illustrates the case $W^{2}>V^{2}$, we can see that there occur many remarkable crossings of the eigenvalues among different levels and series as the strength of $W$ is increased compared to that of $V$. The crossings, however, occur at stronger interaction regimes as the ratio $W / V$ increases. This is a very interesting phenomenon and suggests the existence of a new phase transition. Such a phenomenon of crossings cannot be found in the case of $W^{2}<V^{2}$. 


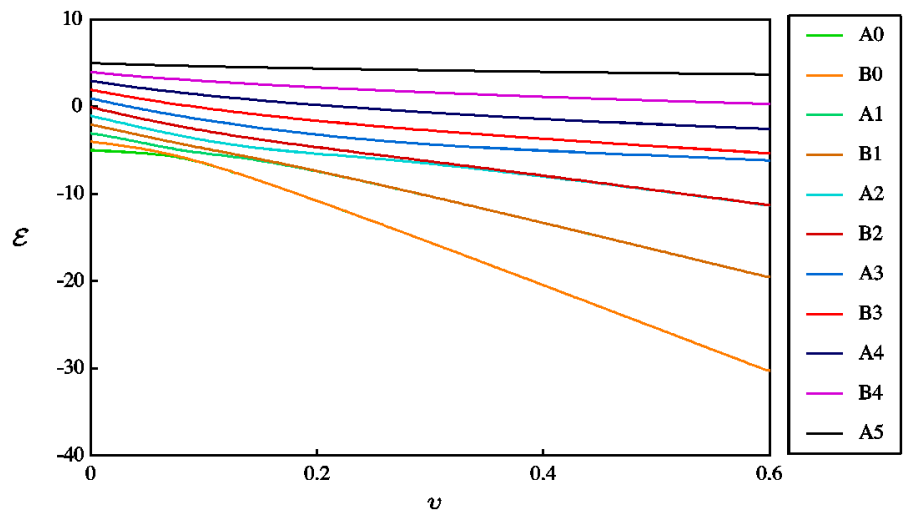

Fig. 1. $\mathcal{E}$ as a function of the parameter $v$ for 10 particles, $W=V$.

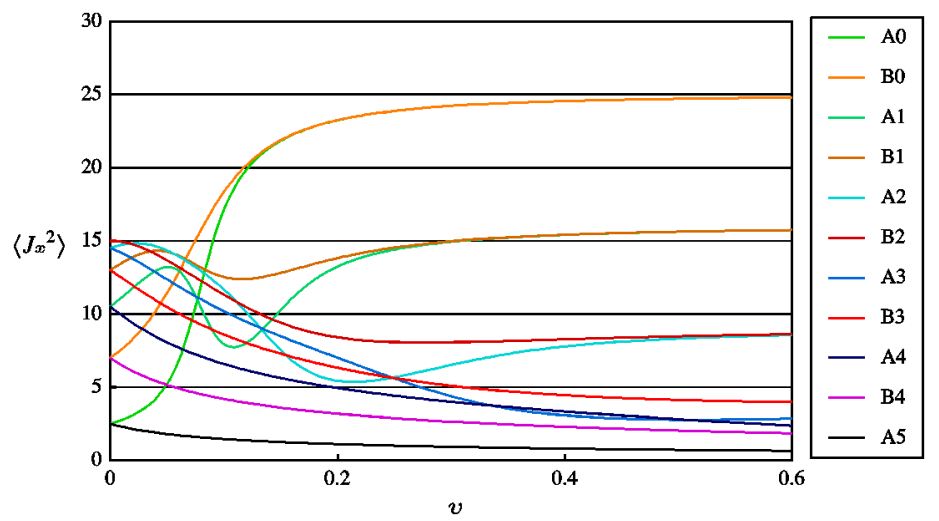

Fig. 2. $\left\langle J_{x}^{2}\right\rangle$ as a function of the parameter $v$ for 10 particles, $W=V$.

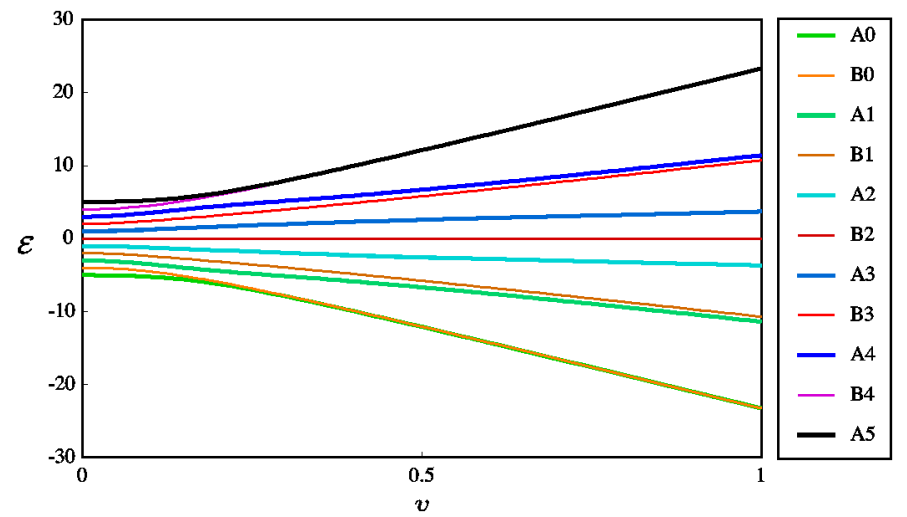

Fig. 3. $\mathcal{E}$ as a function of the parameter $v$ for 10 particles, $W=0$. 


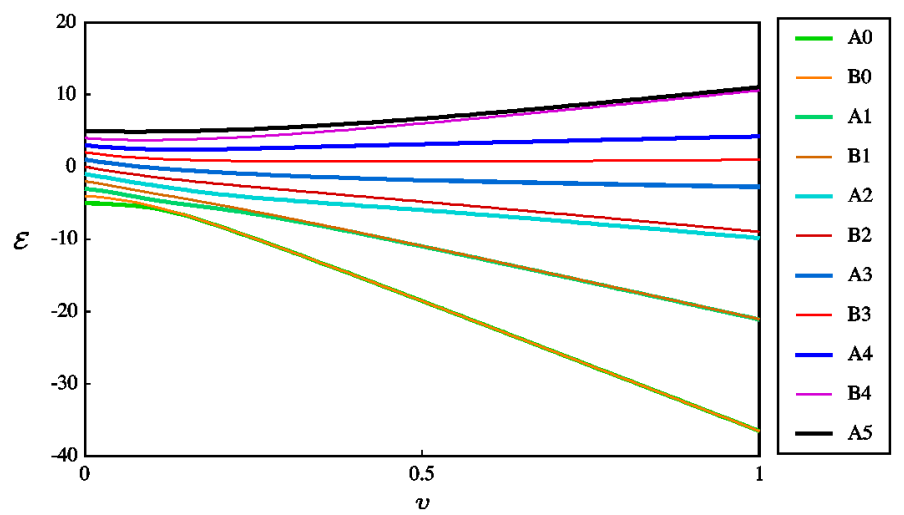

Fig. 4. $\mathcal{E}$ as a function of the parameter $v$ for 10 particles, $W=0.5 \mathrm{~V}$.

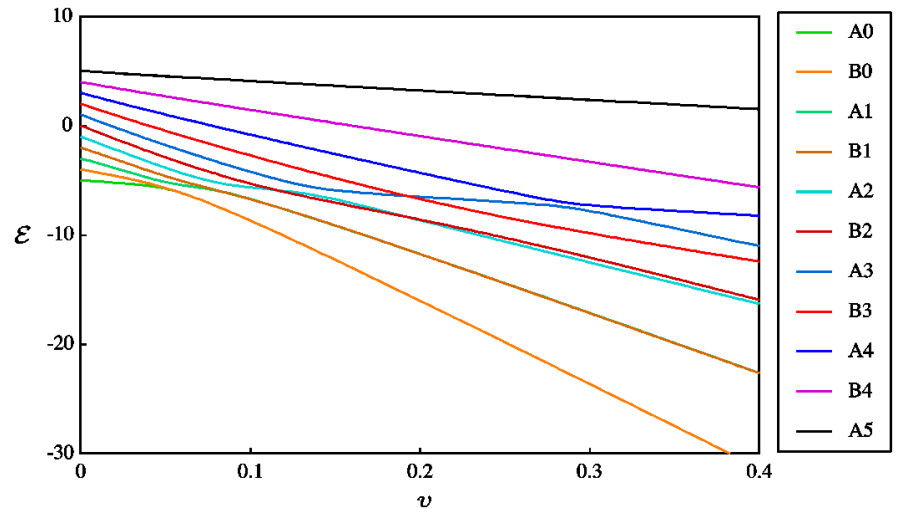

Fig. 5. $\mathcal{E}$ as a function of the parameter $v$ for 10 particles, $W=2 \mathrm{~V}$.

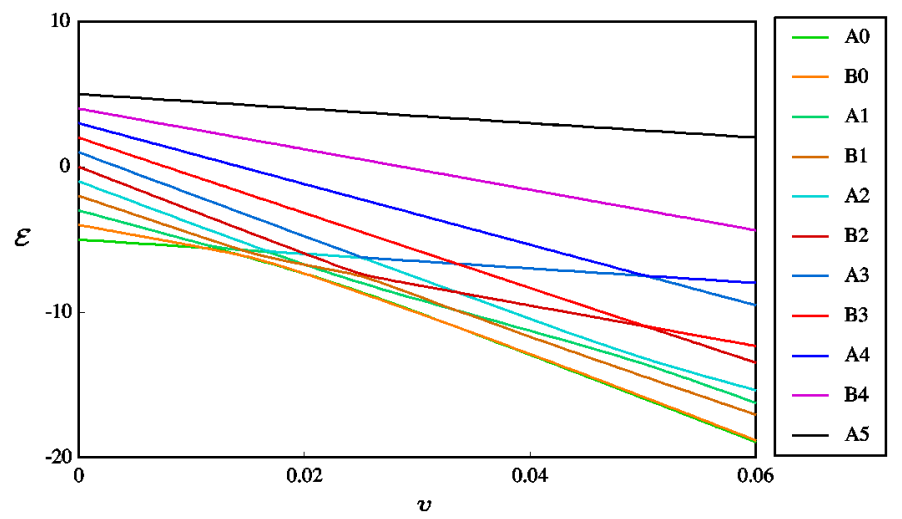

Fig. 6. $\mathcal{E}$ as a function of the parameter $v$ for 10 particles, $W=10 \mathrm{~V}$. 


\section{Discussion and concluding remarks}

The BAE is derived variationally based on the Bethe ansatz. The eigenvalue and eigenstate are given in terms of solutions of the BAE with two parameters.

In the case (1), we adopt the parameters $\beta_{1}$ and $\beta_{2}$ satisfying (3.8). Although, at a glance, the BAE and the equation determining eigenvalues seem to depend on the parameters, the same eigenvalue and the same eigenstate can be obtained for any choice of the parameters only, provided they are subjected to (3.8). It means that the BAE and the equation for the eigenvalue corresponding to each choice of the parameters are transformed to each other by certain transformations of some variables. In relation to this transformation, in (3.7) instead one can put $\Lambda_{j}^{1}$ and $\Lambda_{j}^{2}$ into quite different forms, respectively. As an illustration, for the case of $W=0$ and for the A-series with $n=$ even, if one puts them as

$$
\Lambda_{j}^{1}=\frac{\gamma_{1}}{\epsilon-x_{j}}, \quad \Lambda_{j}^{2}=\frac{i \gamma_{1}}{\epsilon+x_{j}},
$$

with a non-zero $c$-number $\gamma_{1}$ and adopt the assumption

$$
E=-i \epsilon V\left\{(n-1)+i \frac{\epsilon}{V}\right\} \sum_{j} \frac{1}{x_{j}},
$$

another form of the BAE is derived as

$$
\left\{(n-1)+i \frac{\epsilon}{V}\right\} \frac{1}{x_{j}}+\frac{1}{\epsilon-x_{j}}-\frac{1}{\epsilon+x_{j}}=-\sum_{k(k \neq j)} \frac{4}{x_{k}-x_{j}} .
$$

The above equation for the eigenvalue and the BAE coincides with (3.9) and (3.11), respectively when the parameters $\beta_{1}$ and $\beta_{2}$ are taken as $\beta_{1}^{2}=1 / \epsilon$ and $\beta_{2}=i \beta_{1}$.

For the case (2), the BAE

$$
\pm b_{1} \frac{\epsilon}{V}+\frac{b_{1}}{1-b_{1} x_{j}}-\frac{b_{1}}{1+b_{1} x_{j}}=-\sum_{k(k \neq j)} \frac{4}{x_{k}-x_{j}},
$$

is transformed, through the replacements of the variables, $x_{j} \rightarrow 1 / x_{j}$ and $b_{1} \rightarrow 1 / \varepsilon$, into

$$
\mp \frac{1}{V} \frac{1}{x_{j}}+\frac{1}{1-\epsilon x_{j}}+\frac{1}{1+\epsilon x_{j}}=-\sum_{k(k \neq j)} \frac{4 x_{k}}{x_{k}-x_{j}},
$$

a form which is suitable to be compared with the equation of PD [10].

In the case (2) of $W=V$, we have shown that a part of the solutions of the BAE in the strong coupling limit of $v \rightarrow \infty$ is given as the solutions of the zeros of the Sonine polynomial. The BAE in all the regimes of $v$ can be solved easily by the following way: First solve the BAE for a large $v$ adopting the solutions in the limit of $v \rightarrow \infty$ as initial values, and next repeatedly solve the BAE for a smaller $v$ than the former one adopting the solutions obtained formerly as new initial values.

The BAE becomes at present of increasing interest in wider fields of quantum many-body physics. It very often happens that it is difficult to implement some standard diagonalization procedure for a particular matrix, in spite of its diagonalization being in principle possible. Therefore, an exploration into the exact and analytical solutions of the BAE is not only of increasing theoretical interest but also of wider applicability for practical use. 


\section{Acknowledgements}

One of the authors (S.N.) would like to express his sincere thanks to Professor Alex H. Blin for kind and warm hospitality extended to him at the Centro de Física Teórica, Universidade de Coimbra. This work was supported by the Portuguese Project POCTI/FIS/451/94. S.N. was supported by the Portuguese program POCTI/FIS/451/94. One of the authors (S.N.) is greatly indebted to M.C. Ruivo, C. da Providência, M. Fiolhais and C.A. de Sousa for their careful reading of the original manuscript and critical comments.

\section{Appendix A. Solutions for the case $n=$ odd; A-series and B-series}

From (2.4), we obtain the same Eq. (3.2) but with $n^{\prime \prime \prime}$ instead of $n^{\prime}$ and (3.16) but with $n^{\prime \prime \prime}$ instead of $n^{\prime \prime}$. Substitution of the equations into (3.5) leads to

$$
\begin{aligned}
& \mathrm{A}: \quad 0=\sum_{P} P\left[\left[\left\{-\left(n^{\prime}-m^{\prime}\right) \epsilon+m^{\prime} \epsilon-E-W m(n-m)\right\} \Lambda_{P_{m^{\prime}}}^{1} \Lambda_{P_{m^{\prime}+1}}^{2}\right.\right. \\
& -V\left(2 m^{\prime}+1\right)\left(n^{\prime \prime \prime}-m^{\prime}\right) \Lambda_{P_{m^{\prime}}}^{1} \Lambda_{P_{m^{\prime}+1}}^{1} \\
& \left.-V m^{\prime}\left\{2\left(n^{\prime \prime \prime}-m^{\prime}\right)+3\right\} \Lambda_{P_{m^{\prime}}}^{2} \Lambda_{P_{m^{\prime}+1}}^{2}\right] \\
& \times \prod_{\mu \neq P_{m^{\prime}}}^{\mu} \Lambda_{\mu}^{1} \prod_{\substack{v^{\prime \prime \prime} \neq P_{m^{\prime}+1}^{v^{\prime \prime \prime}} \\
v^{\prime \prime \prime}}}^{2} \Lambda^{2} \\
& \mathrm{~B}: \quad 0=\sum_{P} P\left[\left[\left\{-\left(n^{\prime}-m^{\prime}\right) \epsilon+m^{\prime} \epsilon-E-W m(n-m)\right\} \Lambda_{P_{m^{\prime \prime}}}^{1} \Lambda_{P_{m^{\prime \prime}+1}}^{2}\right.\right. \\
& -V\left(2 m^{\prime \prime}+3\right)\left(n^{\prime \prime \prime}-m^{\prime \prime}\right) \Lambda_{P_{m^{\prime \prime}}}^{1} \Lambda_{P_{m^{\prime \prime}+1}}^{1} \\
& \left.-V m^{\prime \prime}\left\{2\left(n^{\prime \prime \prime}-m^{\prime \prime}\right)+1\right\} \Lambda_{P_{m^{\prime \prime}}}^{2} \Lambda_{P_{m^{\prime \prime}+1}}^{2}\right] \\
& \left.\times \prod_{\substack{\mu^{\prime \prime} \\
\mu^{\prime \prime} \neq P_{m^{\prime \prime}}}} \Lambda_{\mu^{\prime \prime}}^{1} \prod_{v^{\prime \prime \prime \prime} \neq P_{m^{\prime \prime}+1}^{v^{\prime \prime \prime \prime}}} \Lambda_{v^{\prime \prime \prime \prime}}^{2}\right] .
\end{aligned}
$$

The case (1) of $W \neq \pm V$.

Using Eq. (3.7) and the assumptions (3.8) and

$$
\left.\begin{array}{l}
\mathrm{A}: \quad E=-\frac{\beta_{1}^{2}+\beta_{2}^{2}}{\beta_{1}^{2}-\beta_{2}^{2}} n^{\prime \prime \prime} \epsilon-\frac{1}{2} \epsilon+\frac{\beta_{2}}{\beta_{1}} n^{\prime \prime \prime} V+\left\{\frac{1}{\beta_{1} \beta_{2}}(n-1) V+\frac{2}{\beta_{1}^{2}-\beta_{2}{ }^{2}} \epsilon\right\} \sum_{j=1}^{n^{\prime \prime \prime}} \frac{1}{x_{j}}, \\
\mathrm{~B}: \quad E=-\frac{\beta_{1}{ }^{2}+\beta_{2}{ }^{2}}{\beta_{1}{ }^{2}-\beta_{2}{ }^{2}} n^{\prime \prime \prime} \epsilon+\frac{1}{2} \epsilon+\frac{\beta_{1}}{\beta_{2}} n^{\prime \prime \prime} V+\left\{\frac{1}{\beta_{1} \beta_{2}}(n-1) V+\frac{2}{\beta_{1}^{2}-\beta_{2}{ }^{2}} \epsilon\right\} \sum_{j=1}^{n^{\prime \prime \prime}} \frac{1}{x_{j}},
\end{array}\right\}
$$

then, the BAEs for $j=1, \ldots, n^{\prime \prime \prime}$ become

$$
\mathrm{A}(\mathrm{B}): \quad\left\{(n-1)+\frac{2 \beta_{1} \beta_{2}}{\beta_{1}^{2}-\beta_{2}^{2}} \frac{\epsilon}{V}\right\} \frac{1}{x_{j}}+\frac{\beta_{1}^{2}\left(3 \beta_{2}^{2}\right)}{1-\beta_{1}^{2} x_{j}}+\frac{3 \beta_{2}^{2}\left(\beta_{2}^{2}\right)}{1-\beta_{2}^{2} x_{j}}=-\sum_{k=1(k \neq j)}^{n^{\prime \prime \prime}} \frac{4}{x_{k}-x_{j}} \text {. }
$$

The case (2) of $W= \pm V$.

Using Eq. (3.7) and the assumptions (3.12) and

$$
\text { A (B): } \quad E=\mp \frac{V}{2} n^{2} \pm \frac{V}{2} n-(+) \frac{1}{2} \epsilon+\epsilon b_{1} \sum_{j=1}^{n^{\prime \prime \prime}} x_{j}
$$


then, the BAEs for $j=1, \ldots, n^{\prime \prime \prime}$ become

$$
\text { A (B): } \quad \pm b_{1} \frac{\epsilon}{V}+\frac{b_{1}\left(3 b_{1}\right)}{1-b_{1} x_{j}}+\frac{3 b_{2}\left(b_{2}\right)}{1-b_{2} x_{j}}=-\sum_{k=1(k \neq j)}^{n^{\prime \prime \prime}} \frac{4}{x_{k}-x_{j}}
$$

\section{References}

[1] J. Bardeen, L.N. Cooper, J.R. Schrieffer, Phys. Rev. 108 (1957) 1175.

[2] R.W. Richardson, Phys. Lett. 3 (1963) 277.

[3] R.W. Richardson, N. Sherman, Nucl. Phys. 52 (1964) 221.

[4] H. Bethe, Z. Phys. 71 (1931) 205;

R.W. Richardson, J. Math. Phys. 6 (1965) 1034.

[5] M. Gaudin, J. Phys. 37 (1976) 1087;

E.K. Sklyanin, Lett. Math. Phys. 47 (1999) 275.

[6] M.C. Cambiaggio, A.M.F. Rivas, M. Saraceno, Nucl. Phys. A 624 (1997) 157.

[7] G. Sierra, Nucl. Phys. B 572 (2000) 517.

[8] J. von Delft, D.C. Ralpf, Phys. Rep. 345 (2001) 61.

[9] H.J. Lipkin, N. Meshkov, A.J. Glick, Nucl. Phys. 62 (1965) 188.

[10] F. Pan, J.P. Draayer, Phys. Lett. B 442 (1998) 7;

F. Pan, J.P. Draayer, Phys. Lett. B 451 (1999) 1.

[11] P. Ring, P. Schuck, The Nuclear Many-Body Problem, Springer, Berlin, 1980.

[12] S. Nishiyama, Nucl. Phys. A 576 (1994) 317.

[13] E.A. Yuzbashyan, A.A. Baytin, B.L. Altshuler, cond-mat/0305635.

[14] B.S. Shastry, A. Dhar, J. Phys. A 34 (2001) 6197.

[15] H. Fukutome, Prog. Theor. Phys. 80 (1988) 417. 